\title{
What features of a nutrition resource are important to adolescents of a low socioeconomic status?
}

\author{
HC Scott ${ }^{1}$, C Craddock ${ }^{2}$ and LCA Craig ${ }^{1, *}$ \\ 'Institute of Applied Health Sciences, University of Aberdeen, Foresterhill, Aberdeen AB25 2ZD, UK: ${ }^{2}$ Communication \\ Design Department, Gray's School of Art, The Robert Gordon University, Aberdeen AB10 7BQ, UK
}

Submitted 18 June 2018: Final revision received 29 September 2019: Accepted 22 0ctober 2019: First published online 27 April 2020

\begin{abstract}
Objective: To identify the key features of a nutrition resource that are important to adolescents of a low socioeconomic status (SES).

Design: Structured interviews were conducted to explore participants' preferences relating to the features of a nutrition resource. Thematic framework analysis was used to determine key themes, subthemes and concepts from the data.

Setting: Streetsport activity sessions, north-east of Scotland.

Participants: Eighteen adolescents aged 12-17 years from a low socioeconomic background.

Results: The overarching themes identified were barriers and facilitators to engagement with a nutrition resource. Adolescents expressed a preference for an app, and this was mainly attributed to convenience and low cost. There was also an emphasis on the integral role social media has in their lives. Aesthetics was a facilitator for both male and female participants, with a particular focus on weight loss. Behaviour change support, including reminders, access to simple recipes and adopting a 'small change approach', were identified as possible facilitators, whereas cost, environmental influences, and existing eating habits were identified as possible barriers to engaging with a nutrition resource.

Conclusions: A number of subthemes, including aesthetics, cost and convenience, which have previously been reported in adolescents with a higher SES, were prominent in our research. The present study contributes to insights relating to behaviour change tools that should be considered when developing a nutrition resource targeting disadvantaged adolescents. Further research focusing on how mobile phone technology and social media can be utilised to support dietary behaviour change in low SES adolescents is recommended.
\end{abstract}

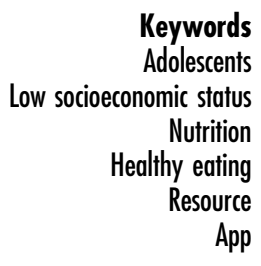

During adolescence, independence is established, and this is often accompanied by changes in eating practices ${ }^{(1)}$. With this, just $8 \%$ of UK adolescents are achieving the recommended five portions of fruit and vegetables per day ${ }^{(2)}$, and the most commonly purchased foods in this age group include confectionary and sugar-sweetened beverages ${ }^{(3)}$. This is of particular concern because dietary habits established during adolescence often continue into adulthood ${ }^{(4)}$, and poor diet is strongly related to chronic diseases, including diabetes, cancer and $\mathrm{CVD}^{(5)}$.

It is well documented that those of a lower socioeconomic status (SES) have a higher risk of poor nutrition than their wealthier peers ${ }^{(6)}$. Those of a low SES typically consume more discretionary foods and less fruit and vegetables $^{(7)}$. These poor food choices parallel health inequalities, which are highlighted by the existence of significant socioeconomic differences in the rates of obesity and chronic diseases ${ }^{(8)}$. In Scotland, children and adolescents in the most deprived areas have a greater risk of obesity in comparison to their age-matched peers in the least deprived areas ${ }^{(9)}$, and this translates to 22 and $13 \%$, respectively. Additionally, the NHS have reported that $26 \%$ of 15 -year-olds from the most deprived areas have extensive tooth decay compared to $12 \%$ of 15 -year-olds who are not eligible for free school meals ${ }^{(10)}$. Consequently, there is a threefold increased risk in adult periodontal disease and caries in low $v$. high childhood

There have been multiple policies introduced to address poor nutrition in children and adolescents in Scotland. SES groups ${ }^{(11)}$. 
Schools are now legally bound to meet minimum nutrition standards ${ }^{(12)}$, while health education and promotion is regarded as mandatory ${ }^{(13)}$. Nonetheless, 33\% of Scottish adolescents aged $12-15$ years remain at risk of overweight and obesity ${ }^{(14)}$. This figure has fluctuated since 1998, but there has not been significant reductions in the proportion of children and adolescents who are at risk of overweight (including obesity), and socioeconomic discrepancies relating to diet continue ${ }^{(15)}$. Published literature supports the theory that increased knowledge is not sufficient to change behaviour $^{(16)}$, as demonstrated in a Scottish study that found that an education intervention increased knowledge but failed to elicit dietary behaviour change in school-aged children $^{(17)}$.

The use of technology-based health promotion is increasing among adolescents, and this has been referred to as a 'new channel' to illicit health-related behaviour change ${ }^{(18)}$. Given the increased reliance on the internet in the adolescent population, there is a need to determine how healthy eating behaviour can be promoted using online tools ${ }^{(19)}$. Research conducted in the USA has demonstrated effective behaviour change for both diet and physical activity in adolescents following on from interventions with an online resource $e^{(20,21)}$. Nonetheless, there is limited research in this area in the UK. The current study sought to investigate the key features of a nutrition resource that are important to adolescents of a low SES, and also aimed to determine if electronic media is an acceptable format for delivering nutrition information to this group.

\section{Methods}

The present study partnered with the Denis Law Legacy Trust, a not-for-profit charity operating in Aberdeen City, north-east Scotland ${ }^{(22)}$, providing a range of free sport (e.g. football, basketball, tennis, netball, dance, street games based on demand) and creative activity sessions to children and adolescents in deprived areas as part of the Streetsport initiative. These work on a pop-up basis, with Streetsport bringing equipment to different sites across the city. Young people participate in a drop-in fashion based on word of mouth, advertising via social media, posters, leaflets, website, local community groups or referral via school or social workers. The research was conducted across three Streetsport sites in Aberdeen City: Northfield, Torry and George Street. These locations are classified within the top $20 \%$ most deprived areas in Scotland in accordance with the Scottish Index of Multiple Deprivation (SIMD) ${ }^{(23)}$, the Scottish Government's tool for identifying areas of deprivation based on postcode.

A review of literature and a series of discussions between the research team and stakeholders with relevant experience, including public health, education and academic professionals as well as Streetsport staff, informed the development of structured interview questions. The researcher also volunteered at Streetsport for 8 weeks prior to data collection, enabling them to gain familiarity with the adolescents and vice versa, establishing a rapport and trust, which is considered a suitable approach for hard-to-reach groups $^{(24)}$.

Recruitment took place at Streetsport sessions. One week prior to data collection, potential participants were approached by the researcher (HS) who explained the study and provided them with an information sheet. Those under the age of 16 were also provided with an information pack for their parents.

In order to participate in the study, those aged 12-15 years were required to return a signed parental consent form. Prior to data collection, participants were asked to complete an assent form. In line with the age of ethical consent in Scotland, those aged 16-17 years were asked to give signed consent themselves before taking part.

Data collection took place in May 2017 and June 2017. Structured face-to-face interviews were conducted by HS at Streetsport sessions. This was seen as the most appropriate location and method of data collection because low response rates are a particular challenge in this group ${ }^{(25)}$, and reducing participant burden was prioritised. The interviews encompassed five main topics outlined in Table 1. Questions were typically open-ended with visual prompts to support (online Supplementary data). This method was chosen to elicit in-depth views from adolescents. However, due to the setting, audio-recording was not possible, and therefore all answers were written down by the researcher. SES was determined using SIMD $2016^{(23)}$.

Data were analysed using a 'Framework' approach, as described by Spencer et al. ${ }^{(26)}$, allowing for comparisons in responses across subgroups. The responses were read by two researchers (HS, an MSc Human Nutrition student with interest in health and fitness; and LCAC, a researcher with a background in health sciences and public health nutrition) who identified themes and subthemes reflecting all the responses, and met to discuss their independent analysis of the data. Participant responses were coded against the subthemes using Microsoft Excel.

\section{Results}

A total of fifty-five participants were given information sheets during the recruitment phase: twenty-two aged 16-17 years, and twenty-three under the age of 16 . Eighteen adolescents participated in this study (33\%); ten males and eight females, the majority of whom ( $n$ 16) were aged 16-17 years and most of them ( $n$ 14) lived in most deprived SIMD areas 1 and 2 (see Table 2 for detailed demographics). Five participants were volunteers at Streetsport, but had previously been participants and wanted to continue attending activity sessions. 
Table 1 Description of topics discussed during structured interviews

\begin{tabular}{|c|c|}
\hline Topic area & Focus of questions \\
\hline Background information & $\begin{array}{l}\text { Understanding of the term 'healthy eating' } \\
\text { Interest in learning more about 'healthy eating' } \\
\text { Previous resources used to look up information on healthy eating }\end{array}$ \\
\hline Format of tool & $\begin{array}{l}\text { Preference in regard to the delivery of healthy eating information } \\
\text { Reasons behind preferred choice }\end{array}$ \\
\hline Content & $\begin{array}{l}\text { Goal-setting } \\
\text { Food diaries } \\
\text { Reminders } \\
\text { Celebrity endorsement } \\
\text { Quiz } \\
\text { Food swaps } \\
\text { Topics of interest }\end{array}$ \\
\hline $\begin{array}{l}\text { Barriers and facilitators } \\
\text { Participant input }\end{array}$ & $\begin{array}{l}\text { Any specific barriers or motivators to using a nutrition resource } \\
\text { Any additional ideas that participants felt would be important for a healthy eating resource }\end{array}$ \\
\hline
\end{tabular}

Table 2 Participant demographic information recorded at structured interviews

\begin{tabular}{llllcl}
\hline ID & Age & Gender & $\begin{array}{c}\text { Location of data } \\
\text { collection }\end{array}$ & $\begin{array}{c}\text { SIMD 2016 } \\
\text { quintile* }\end{array}$ & Role at Streetsport \\
\hline P01 & 17 & Male & Torry & 3 & Volunteer \\
P02 & 17 & Female & Torry & 2 & Volunteer \\
P03 & 16 & Male & Torry & 2 & Volunteer \\
P04 & 16 & Female & George St & 2 & Participant \\
P05 & 17 & Female & George St & 2 & Participant \\
P06 & 13 & Female & George St & 2 & Participant \\
P07 & 16 & Female & Northfield & 3 & Participant \\
P08 & 16 & Female & Northfield & 1 & Volunteer \\
P09 & 16 & Female & Northfield & 1 & Participant \\
P10 & 16 & Female & Northfield & 2 & Participant \\
P11 & 17 & Male & Northfield & 3 & Participant \\
P12 & 16 & Male & Torry & 1 & Participant \\
P13 & 17 & Male & Torry & 2 & Participant \\
P14 & 13 & Female & Torry & 1 & Participant \\
P15 & 16 & Male & Northfield & 5 & Participant \\
P16 & 16 & Female & Northfield & 1 & Participant \\
P17 & 17 & Male & Northfield & 1 & Volunteer \\
P18 & 16 & Male & Torry & 2 & Participant \\
\hline
\end{tabular}

*1 = most deprived, 5 = least deprived.

The overarching themes identified were barriers and facilitators to engaging with a healthy eating resource. Emergent subthemes were identified along with key concepts, illustrated in Fig. 1. Cost and convenience were both prominent emerging subthemes considered as both barrier and facilitator. Barriers identified included existing unhealthy eating habits and environmental influences, whereas facilitators included aesthetics, health and fitness, communication with peers, behaviour change, developing knowledge of healthy eating and additional engaging features. These emerged from the researchers' interpretation of the data in the context of the aims of the study and were partly driven by the questions asked.

\section{Cost}

Cost was identified as a prominent emerging subtheme linked to both barriers and facilitators. Providing a resource that is free was important to participants, while paying to access a nutrition tool was not considered a priority:

'There are other resources that are free so why spend money when you don't have to.' (P02, female, 17 years)

Participants perceived healthy foods as expensive, whereas less nutritious foods were considered cheap. There was reference to the cost of food being a barrier to engaging with a nutrition tool because this factor alone strongly influences their current food choices:

'The cost of healthy food is expensive. Salad v. burger in McDonalds. I always go for the cheaper option'. (P08, female, 16 years)

Additionally, participants felt that the cost of healthy food prevented them from relating to famous role models because they are wealthier and can afford healthier foods: 


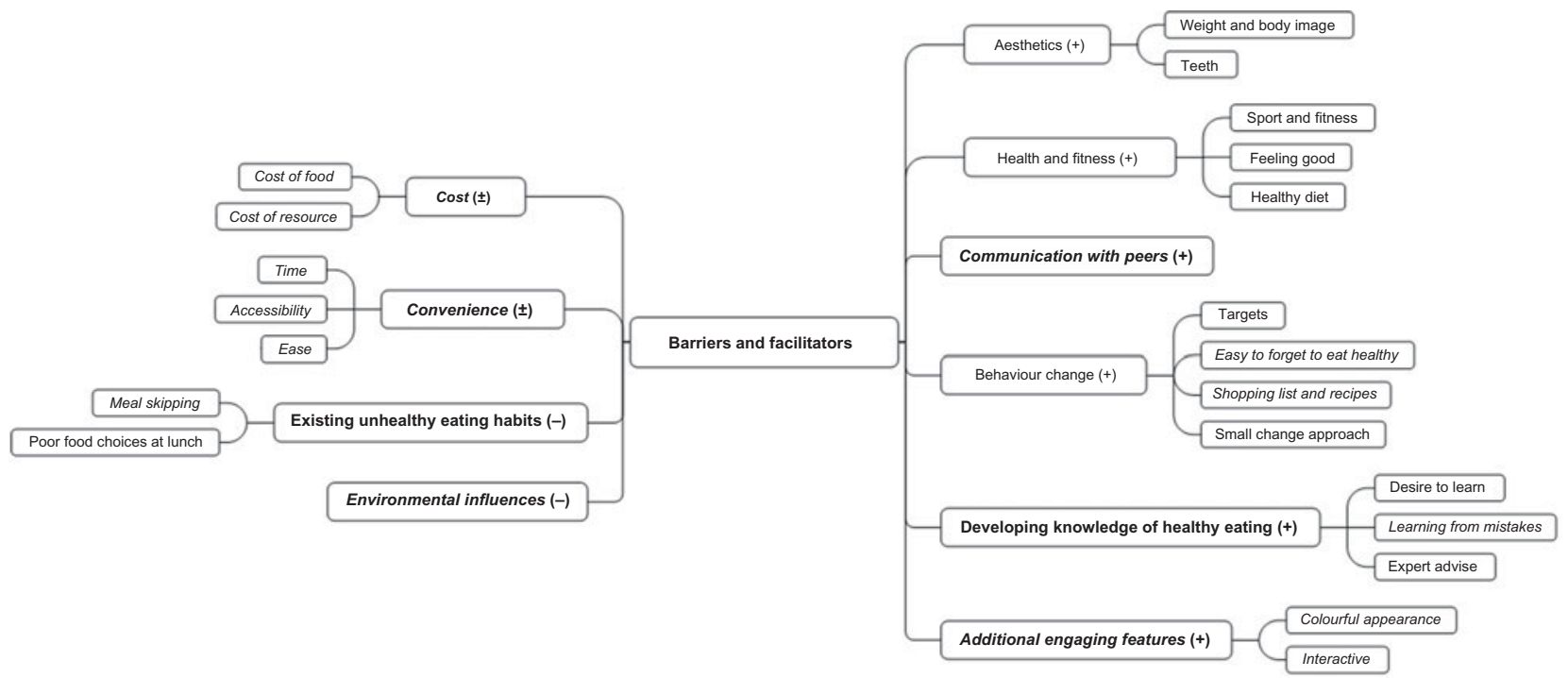

Fig. 1 A summary of themes, subthemes and concepts that were identified from the thematic analysis of responses from structured interviews. + and - represent facilitators and barriers, respectively, whereas \pm represents subthemes that were identified as both a barrier and facilitator to engaging with a nutrition resource. Bold text identifies prominent subthemes; and italics, emerging subthemes

'They (celebrities) have money to eat better and to have personal trainers and I don't. Healthy food is expensive'. (P05, female, 17 years)

On the other hand, participants discussed what they liked most about the apps they are currently using. Free access was an important feature, and therefore cost was also identified as a facilitator to engaging this group with a nutrition resource:

'They (apps) are a free way to speak to folk.' (P15, male, 16 years)

\section{Convenience}

One of the most prominent subthemes to emerge was convenience, with time, accessibility and ease identified as important concepts. When asked if there was anything that would prevent participants from using a healthy eating resource, there was an emphasis that time is a barrier, with other things taking priority:

'I go to work early and come back late. I don't have time to read stuff on healthy eating'. (P15, male, 16 years)

However, this was not just the case for older participants; indeed, one of the participants who was under the age of 16 said 'I don't have a lot of free time', and for this reason she reported that she would not engage with a healthy eating app, but instead she would prefer an interactive learning session. However, this was not the reported preference for the other participant under the age of 16 . Additionally, when discussing the content of a nutrition tool, it was common for participants to mention that time was a limiting factor and that they would not want anything that 'takes too long'.

On the other hand, time was also identified as a facilitator, and this was apparent when participants described an app as their preferred format for a nutrition tool:

'Apps are quick and you can use them at any time.'

(P03, male, 16 years)

The accessibility of apps and the ability to use them anywhere at any time was also a significant feature that participants made reference to, and was identified as both a barrier and facilitator to engaging participants with a nutrition tool. This response was typical:

'An app would be good because you have your phone on you all the time and can use it whenever or wherever.' (P04, female, 16 years)

On the other hand, the majority of those who did not choose an app as a preferred method made reference to having limited data storage on their phones, which would make an app less accessible. These participants had a preference to using either a website or television as a source of nutrition information, and again ease of access was a facilitator:

'Websites are easy to access, apps take up storage.' (P03, male, 16 years)

'I watch TV every night. It doesn't need effort.'

(P15, male, 16 years)

When asked what participants liked about the apps they are currently using, they described them as 'easy to use' and 'easy to look at', and it was apparent that using their phones was effortless: 
'My phone is always with me. It's easier, I can use 4G wherever I am.' (P08, female, 16 years)

Features such as taking photographs of foods eaten were also viewed as an easy option and were considered an appealing feature to some participants:

'Taking photos would be easier. My phone is always on me and I can take photos wherever.' (P10, female, 16 years)

However, there were mixed views on this, and those who did not want to take photographs of their food also spoke about ease, with one participant saying 'it's easier to write'.

\section{Existing unbealthy eating habits}

Existing unhealthy eating habits were mentioned throughout the interviews, and there was a general consensus that there are 'lots of unhealthy options' available which could prevent participants from engaging with a nutrition tool. Meal skipping, in particular breakfast, was discussed:

'It's the only time I eat during the day (lunch). I don't

eat breakfast'. (P02, female, 17 years)

Moreover, it was common for participants to make reference to their poor food choices during their lunch break. Consequently, there was an interest in learning more about how to make healthier food choices at lunch time. This example was typical:

'I normally eat bad at lunch, there are lots of unhealthy options and I never think to have healthy food.' (P09, female, 16 years)

\section{Environmental influences}

Participants acknowledged that the environment encourages them to purchase unhealthy foods, and this was seen as a barrier that may prevent them from engaging with a healthy eating resource. One participant felt that 'the food around you stops you focusing on being healthy'. In agreement with this, advertisements were also considered a barrier:

'You see a new food and immediately want to try it even when it's unhealthy. Even if you don't want to eat bad seeing the advert will make you want to try it.' (P03, male, 16 years)

\section{Aestbetics}

Aesthetics was a prominent subtheme and was considered a strong motivator for participants when discussing engagement with a nutrition tool. Weight and body image were frequently referenced, and this was common in both male and female respondents:

'I feel better when I lose weight, I have more friends when I am thinner.' (P10, female, 16 years)
This focus on body image was also apparent when participants were asked about their thoughts relating to the use of celebrities as a role model for healthy eating:

'If a celebrity had a nice body this would motivate me. I want a nice body, I would focus on weight.'

(P04, female, 16 years)

In addition to body image, participants made reference to how their teeth look. It was viewed as important to not have 'rotten teeth', and making healthier choices, such as choosing sugar-free drinks, was mentioned. Participants reported that they would want to learn more about how food affects your teeth:

'It looks good to have healthy teeth and a nice smile.' (P08, female, 16 years)

\section{Health and fitness}

Throughout the interviews, health and fitness was commonly discussed, with sport, feeling good and having a healthy diet identified as the main concepts. Improving fitness was important and was a prominent subtheme that emerged during interviews with male participants, and was occasionally referenced in the responses of female participants:

'Healthy eating would help to run well for football.'

(P08, female, 16 years)

'I would want to learn more to get better at rugby and football.' (P17, male, 17 years)

Additionally, it was common for participants to make reference to the relationship between a healthy diet and feeling good, and this was viewed as a motivator to improving food choices:

'If you feel good from eating better you would stick to it, otherwise you wouldn't.' (P15, male, 16 years)

In general, there was a positive attitude towards having a healthy diet among participants, and all but four participants reported that they would be interested in learning more about nutrition. Those who did not want to learn more either attributed this to time and cost, or they felt they already had adequate knowledge of what foods are good for you, with one participant saying 'I already know what to do, eat well to move well'. When discussing food swaps to enable a healthier diet, some participants reported they would choose the healthiest option imminently:

'I would choose fruit because it's the healthiest option so may as well go for that one.' (P12, male, 16 years)

Additionally, some participants reported that they are currently trying to improve their diet:

'I would set healthy eating targets. I did it today. I wanted to have the healthier option today. When I was offered fizzy juice I asked for water.' (P06, female, 13 years) 
However, others preferred to make smaller changes, and this is discussed later under 'behaviour change'.

\section{Communication with peers}

All but one participant reported using some form of social media, which was described as being a 'good way to stay connected'. The participant who did not report currently using social media was under the age of 16 and reported that her phone was broken. There was a strong emphasis on the importance of communicating with friends, and this was an attractive feature of their current apps:

'Apps keep you connected with friends, you can talk to them all the time and see what they are doing.' (P04, female, 16 years)

\section{Behaviour change}

Throughout the discussions, participants inferred that behaviour change support was an important feature of a nutrition resource. This was reported across the age groups and in both males and females. It was apparent that setting nutrition-related targets was viewed as an effective approach:

'That would help (setting healthy eating targets) because if you set targets it will help you with eating healthy in everyday life.' (P18, male, 16 years)

Additionally, reminders related to healthy eating targets were viewed as an important feature of a nutrition resource since it was common for participants to reference healthy eating as something that is 'easy to forget':

'I would like this because it would stop me forgetting. It's easy to forget to eat well.' (P06, female, 13 years)

When asked for their own views on what features of a nutrition tool are important, there was reference made to recipes and shopping lists to support behaviour change. Participants felt that recipes that are easy to prepare would support them in improving their diet:

'There is a lack of simple recipes. Something with few ingredients that is easy to make. Pictures would be best.' (P02, female, 17 years)

Additionally, an example shopping list was also seen as a good tool to support dietary behaviour change:

'A list of what to eat, like a shopping list of healthy foods to buy for a week, to be organised and not just buy whatever.' (P16, female, 16 years)

Finally, adopting a small change approach was identified as a potential facilitator to engaging participants with a nutrition tool. Providing options for snack swaps was discussed, with options ranging from reduced portion sizes to healthier choices. There was a mixed response in what participants would prefer, but it was apparent that still having the option to 'eat what you want' or to 'cut down slowly' was important:
'You don't have to give up what you like, I'd rather cut down.' (P18, male, 16 years)

\section{Developing knowledge of bealthy eating}

There was a positive attitude to expanding knowledge of nutrition-related topics among the group, with access to an education resource being viewed as 'interesting' and 'helpful'. Participants reported that they wanted to learn more about the composition of foods:

'I want to learn what is in different foods and what is good and bad.' (P02, female, 17 years)

Conversations were also led to which topics would be of interest to participants, and increasing knowledge was desirable.

'I would like to know about this (energy balance and weight) to know what to eat.' (P14, female, 13 years)

Although the most commonly reported topic of interest was energy balance and weight, other topics, including how food affects your teeth, making healthy choices at lunch time and increasing fruit and vegetable consumption, were also areas that participants wanted to learn more about.

When discussing the inclusion of a quiz as a learning tool for a nutrition resource, participants generally felt this was an acceptable approach that could support them with their learning:

'You would get feedback which helps with learning and learning from mistakes.' (P02, female, 17 years)

Additionally, expert advice that you can 'trust' is a concept that was important:

'It's experts who are giving advice, they have personal experience and you can trust their advice.' (P11, male, 17 years)

\section{Additional features to support engagement with a nutrition tool}

The appearance of a nutrition resource was important to participants. One participant reported that taking photographs of food is engaging because it would make the resource 'colourful'. Additionally, it was seen that this is a good approach to draw attention to the resource:

'It should be bright coloured to attract people. It needs to be enjoyable. Otherwise you just get bored.' (P11, male, 17 years)

A further concept that was commonly discussed during the interviews was the general dislike of reading, which was perceived as boring and would potentially discourage participants from engaging with a nutrition tool. For this reason, something with an interactive element was considered a facilitator to engaging this group:

'Quizzes would be alright because it's different and better than reading.' (P12, male, 16 years) 


\section{Discussion}

This study points to a range of key barriers and facilitators to engaging adolescents from a low socioeconomic background with a nutrition resource, and the majority of participants identified both barriers and facilitators. The study found that there is an interest in learning more about nutrition within this group, and this was emphasised throughout the interviews, despite the fact that many appeared to already have some knowledge of healthy eating. There was a general preference for a nutrition tool to be in an app format, and this was attributed to convenience and low cost, which is possibly unsurprising given that the research was conducted amongst low SES adolescents. Indeed, mobile phone health-related resources have previously been shown to increase the potential for accessing hard-to-reach groups ${ }^{(27)}$. Nonetheless, one participant who was under the age of 16 reported that she did not have time for her phone. Since this age group was poorly represented in our sample, we are unable to draw conclusions relating to discrepancies between preferences of younger and older adolescents. However, Ofcom have reported that $71 \%$ of adolescents aged $12-15$ years have a social media site, and $70 \%$ of those aged 13 years have a smartphone ${ }^{(28)}$. They also highlighted that no particular socioeconomic group is more or less likely to own a smartphone. Therefore, an app is possibly an acceptable format for a nutrition resource in this group, but further research is required to confirm this among younger adolescents.

It was also of note that our participants valued apps for social interaction, and the only apps participants spoke about were social media. With the knowledge that social norms among peers influence adolescent food choices and are often unsupportive of healthy eating ${ }^{(29)}$, there is an opportunity for social media to be utilised as a tool to support behaviour change. Indeed, a recent qualitative study reported that Australian adolescents from disadvantaged areas favoured electronic media as a method for accessing nutrition information, with frequent reference to Facebook ${ }^{(30)}$. With this, there is a demand for research to focus on how the use of social media can be adapted to promote diet-related behaviour change across the adolescent population.

The importance of convenience was related to both the nutrition tool and the preparation of food, and it was clear that other factors in their life took priority. Indeed, previous research on adolescents has reported that time and accessibility were key themes relating to the use of online sexual health education resources ${ }^{(31)}$. Additionally, research focusing on older adolescents and young adults of a low SES found that participants liked foods that were fast and easy ${ }^{(32)}$, which aligns with the reference made to 'simple' recipes 'with few ingredients' during our interviews. Previous qualitative research has reported that parents of adolescents of a low SES typically do not influence food choices, and family meals are not part of their normal routine, which conflicts with what is reported from those of a higher SES ${ }^{(33)}$. Therefore, recipes provided should be suitable for adolescents, as they are likely to be preparing their own meals, and therefore simplicity is important. There were mixed preferences for use of photographs $v$. written information; however, the use of images could overcome possible literacy issues that may be more common among low SES groups.

Convenience was also linked to the preference for a 'small change approach' among some of the participants. Although many of our participants said they would make healthy changes imminently, and indeed some already were, others reported they would rather reduce portion sizes and make smaller changes towards healthy eating. It is important to recognise that those from lower SES may not have access to healthy food options at home, and it has been reported that those of lower SES purchase significantly lower percentages of energy from fruit and vegetables $^{(34)}$. Therefore, the consumption of the healthiest option may not be easy for adolescents in this group, and providing options that include reducing portion sizes could be more realistic. This approach has been shown to enhance self-efficacy through achieving small lifestyle changes, which has previously been demonstrated as an effective method for eliciting behaviour change in adolescents $^{(35)}$.

Cost was also a prominent subtheme that was identified as both a barrier and facilitator, and it was perceived that healthy diets were expensive. The price of food is one of the main factors that influences food choices in lower SES groups ${ }^{(36)}$, and adolescents typically look for cheap foods ${ }^{(37)}$. Previous studies have demonstrated that subsidising healthy foods increases consumption among the adolescent population( ${ }^{(38)}$. Consequently, it has been recommended that budgeting and demonstrating costeffective options should be covered in nutrition education for lower SES groups ${ }^{(39)}$, and this is an important consideration for the development of a nutrition resource targeting adolescents of low SES. Additionally, the cost of a nutrition tool could be a facilitator or barrier to engaging adolescents, and this is in agreement with the previous research that has reported that low SES adolescents feel that nutrition information should be free ${ }^{(31)}$.

Qualitative research has previously demonstrated that aesthetics is a priority to adolescents ${ }^{(40)}$, and this was a prominent subtheme in our study. Weight loss was frequently mentioned, and this was observed across the age groups, in both males and females. Indeed, adolescents have previously reported that a thin body holds power in both friendships and social groups at school ${ }^{(41)}$. Since dieting behaviours for weight loss are a risk factor for developing unhealthy eating behaviours in adolescents ${ }^{(42)}$, an integrated approach that provides information on energy balance and weight, and the dangers of excessive dieting, could have advantages in terms of relevance and safety ${ }^{(43)}$. Another aesthetical motivator was having healthy teeth. 
Zotti et $a l .{ }^{(44)}$ found that using an app to enhance standard oral hygiene motivation among an adolescent population was effective in improving their oral health status. This intervention included the use of photographs and text messages to motivate and remind participants to adhere to instructions, highlighting the acceptability of an interactive tool to elicit health behaviour change in adolescents.

The association between nutrition and fitness was well recognised among our participants, and this may be due to their current interest in sport. Indeed, published literature focusing on improving nutrition in adolescents has often targeted physical activity and nutrition together ${ }^{(45,46)}$. The idea of using a professional sportsperson as a role model to improve nutrition elicited mixed responses. Knowledge focusing on nutrition for professional athletes was thought to be motivational to those who wanted to improve sport performance, and this was previously proved to be effective in health promotion interventions targeting children and adolescents of low $\operatorname{SES}^{(47)}$. However, during our interviews, it was apparent that the perceived higher cost of healthy foods meant that some participants felt they could not relate to famous role models who have more money available. Again, this highlights the need to consider cost and relevance when designing a nutrition tool to engage adolescents of low SES.

Finally, the results demonstrated that approaches for health behaviour change are viewed as important to our participants, and some were already goal-setting. Interventions targeting low-income groups are advised to include simple techniques such as encouraging participants to set goals that help them translate motivation into action $^{(48)}$, and this approach was well received across the interviews. Interestingly, our participants felt that healthy eating is something they often forget about. Indeed Backett-Millburn et al. have previously discussed that parents of low SES adolescents consider food choices to be the responsibility of their children, and therefore this group may not be prompted to eat healthy foods at home ${ }^{(33)}$. Randomised controlled trials have demonstrated improved efficacy and adherence to health-related interventions that have included reminders, in the form of text messages, among both children and adolescents ${ }^{(49,50)}$. Nonetheless, of the current health apps available to adolescents, reminders are not commonly utilised to support behaviour change ${ }^{(51)}$. This demonstrates the need to address the most effective behaviour change techniques in apps targeting this group.

A number of study limitations should be acknowledged. The approach for obtaining consent meant it was challenging to recruit participants under the age of 16 , and the low response rate highlights this. This limited our ability to draw conclusions for younger adolescents from the results available. Indeed, previous research on adolescents has reported that studies adopting opt-out consent drew higher participation and a more representative sample, particularly among low SES populations ${ }^{(52)}$. It is also possible that the sample was biased towards those interested in healthy eating. There were multiple participants who were engaged with sport, while others had an interest in nutrition that was attributed to career aspirations or previous dieting. Therefore, the results may not be reflective of the wider low SES adolescent population. Due to the drop-in style of the Streetsport sessions, the number of eligible participants was difficult to assess. We did not routinely collect information on whether participants were at school or working, and also whether or not they were living with parents or elsewhere, which may have influenced their responses and limited our ability to interpret the data. Finally, there were multiple distractions, including noise, disruption from peers and adverse weather conditions, at the Streetsport sessions. This made recruitment challenging and prevented the researcher from audio-recording the interviews. Also, being interviewed with their friends nearby may have influenced the adolescents' responses. Although the researcher attempted to capture the words as spoken, it may have influenced the data in the way it was written. With written notes, there is a risk of the interviewer biasing the data collected, by being subjective or misinterpreting what is said. It could be considered that the data had already been partly synthesised and interpreted by the interviewer and was not first-order data. In addition, this approach interrupted the flow of the interview and limited the depth and richness of the data collected. It also limited the second researcher analysing the data from fully understanding the context of the words. Finally, the background of the researchers may have likely influenced the interpretation of the data.

On the other hand, the qualitative design allowed insights to be gained into personal, social and environmental factors relating to engaging this group with a nutrition tool. It was also beneficial for the researcher to volunteer at Streetsport prior to data collection, as this allowed for most appropriate methodologies to be identified and trust to be built with this 'hard-to-reach' group. The perspective of disadvantaged adolescents is poorly represented on this topic, and the information collected provides insightful considerations for the development of a nutrition tool targeting this group.

In summary, the findings align with current research relating to adolescents, mainly conducted among those of higher SES, in terms of their preference to receive health information through electronic media. Convenience, aesthetics and cost are key considerations, and this has previously been reported elsewhere. More specifically, resources targeting this group should be interactive as reading is considered 'boring' and is likely to prevent engagement. The findings provide new insights relating to appropriate behaviour change support, such as access to easy recipes, reminders and a small change approach, which were identified as facilitators to engaging adolescents of low SES with a nutrition resource. More research is required to determine if there are discrepancies between younger and older adolescents, 
as well as to establish how social media can be utilised to encourage dietary behaviour change in this group.

\section{Acknowledgements}

Acknowledgements: The authors would like to thank Dr Mary Bellizzi, Dr Julia Allan, Julia Clark, Karen Tosh, Dr Heather Morgan and Neil Hendry for their contributions during the development of structured interview questions. We would like to thank Hannah Clews for her continued support during the Streetsport sessions, both prior to and during data collection. Additionally, we would like to thank the staff at Streetsport for their feedback on structured interview questions. Financial support: This work was supported by the University of Aberdeen. Conflicts of interest: There are no conflicts of interest. Authorship: HS made substantial contributions to the background research, development of interview questions, project design, obtaining ethical approval, recruitment, data collection, data analysis and preparation of this article. CC initiated this project and supported with the development of interview questions and review of this paper. LCAC made substantial contributions to the background research, development of interview questions, project design, obtaining ethical approval, data analysis and review of the article. Ethics of human subject participation: This study was conducted according to the guidelines laid down in the Declaration of Helsinki, and all procedures involving human subjects were approved by the University of Aberdeen Rowett Ethics Review Panel. Written informed consent was obtained from all subjects.

\section{Supplementary material}

For supplementary material accompanying this paper visit https://doi.org/10.1017/S1368980019004476

\section{References}

1. Story M, Neumark-Sztainer D \& French S (2002) Individual and environmental influences on adolescent eating behaviours. J Am Diet Assoc 102, 40-51.

2. Roberts C, Steer T, Maplethorpe N et al. (2018) National Diet and Nutrition Survey. https://www.gov.uk/government/ collections/national-diet-and-nutrition-survey (accessed June 2018).

3. Macdiarmid JI, Wills WJ, Masson LF et al. (2015) Food and drink purchasing habits out of school at lunchtime: a national survey of secondary school pupils in Scotland. Int J Behav Nutr Phys Act 12, 98.

4. Franko DL, Cousineau TM \& Trant M (2008) Motivation, selfefficacy, physical activity and nutrition in college students: randomized controlled trial of an internet based education program. Prev med 47, 369-377.

5. Northstone K, Smith ADAC \& Cribb VL (2013) Dietary patterns in UK adolescents obtained from a dual-source FFQ and their associations with socio-economic position, nutrient intake and modes of eating. Public Health Nutr 17, 1476-1485.

6. Mackenbach JS, Brage S, Forouhi NG et al. (2015) Does the importance of dietary cost for fruit and vegetable intake vary by economic position. Br J Nutr 114, 1464-1470.

7. Masson LF, Bromley C, Macdiarmid JL et al. (2012) Survey of Diet Among Children in Scotland (2010) Volume 1: Diet, Obesity and Physical Activity. Food Standards Agency Scotland. https://www.foodstandards.gov.scot/publicationsand-research/publications/survey-of-diet-among-children-inscotland (accessed April 2020).

8. Maguire ER \& Monsivais P (2015) Socio-economic dietary inequalities in UK adults: an updated picture of key food groups and nutrients from national surveillance data. $\mathrm{BrJ}$ Nutr 113, 181-189.

9. Food Standards Scotland (2016) Diet and Nutrition: Proposal for Setting the Direction for Scottish Diet. http://www. foodstandards.gov.scot/fss-board-meeting-20-january-2016 (accessed February 2017).

10. NHS (2015) Are Half of Children's Teeth Rotten? NHS. http:// www.nhs.uk/news/2015/03March/Pages/are-half-ofchildrens-teeth-rotten.aspx (accessed March 2017).

11. Poulton R, Caspi A, Milne BJ et al. (2002) Association between children's experience of socioeconomic disadvantage and adult health: a life-course study. Lancet 360, 1640-1645.

12. Scottish Government (2008) Healthy Eating in Schools: A Guide to Implementing the Nutritional Requirements for Food and Drink in Schools (Scotland) Regulation 2008. The Scottish Government. http://www.gov.scot/resource/ doc/238187/0065394.pdf (accessed January 2017).

13. Scottish Government (2010) Health and Wellbeing in the Curriculum for Excellence. Scottish Government. http:// www.gov.scot/Topics/Education/Schools/HLivi (accessed March 2017).

14. McLean J, Christie S, Hinchliffe S et al (2018) Scottish Health Survey: 2017 Edition. Scottish Government. http://www.gov. scot/Topics/Statistics/Browse/Health/scottish-health-survey (accessed March 2019).

15. McNeill G, Masson LF, Macdiarmid JI et al. (2016) Socioeconomic differences in diet, physical activity and leisure time screen use among Scottish children in 2006 and 2010: are we closing the gap. Public Health Nutr 20, 951-958.

16. Wardle J, Parmenter K \& Waller J (2000) Nutrition knowledge and food intake. Appetite 34, 269-275.

17. Griffin TL, Jackson DM \& McNeill G (2015) A brief educational intervention increases knowledge of the sugar content of foods and drinks but does not decrease intakes in Scottish children aged 10-12 year. J Nutr Edu Behav 47, 367-373.

18. Cullen KW, Thompson D, Boushey C et al (2013) Evaluation of a web-based program promoting healthy eating and physical activity for adolescents: teen choice: food and fitness. Health Educ Res 28, 704-714.

19. Ritterband LM, Thorndike FP, Cox DJ et al. (2009) A behavior change model for internet interventions. Ann Behav Med 38, $18-27$.

20. Mauriello LM, Ciavatta MH \& Paiva AL (2010) Results of a multi-media multiple behaviour obesity prevention program for adolescents. Prev Med 51, 451-456.

21. Whittenmore R, Jeon S \& Grey M (2012) An internet obesity prevention program for adolescents. $J$ Adolesc Health 52, 439-447.

22. Streetsport (2017) About Streetsport. Introduction. Denis Law Legacy Trust. http://streetsport.org/about/ (accessed May 2017).

23. Scottish Government (2016) SIMD. Scottish Index of Multiple Deprivation. Scottish Government. http://simd.scot/2016/\#/ simd2016/BTTTFTT/9/-4.0000/55.9000/ (accessed June 2017). 
24. Shaghaghi A, Bhopal RS \& Sheikh A (2011) Approaches to recruiting 'Hard To Reach' populations into research: a review of the literature. Health Promot Perspec 1, 86-94.

25. Bonevki B, Randell M, Paul C et al. (2014) Reaching the hardto-reach: a systematic review of strategies for improving health and medical research with socially disadvantaged groups. BMC Med Res Methodol 14, 14-42.

26. Spencer L, Richie J \& O'Connor W (2003) Carrying out qualitative analysis. In Qualitative research practice: a guide for social science students and researchers, pp. 219-262 [J Ritchie \& J Lewis, editors]. London: Sage Publications Ltd.

27. Proudfoot J (2013) The future is in our hands: the role of mobile phones in the prevention and management of mental disorders. Aust N Z J Psychiatry 47, 111-113.

28. Ofcom (2014) Children and Parents: Media Use and Attitudes Report. https://www.ofcom.org.uk/_data/assets/pdf_file/ 0027/76266/childrens_2014_report.pdf (accessed July 2017).

29. Stok MF, Ridder DTD, Vet E et al. (2013) Don't tell me what I should do, but what others do: the influence of descriptive and injunctive peer norms on fruit consumption in adolescents. Br J Health Psychol 19, 52-64.

30. Stephens LD, McNaughton SA, Crawford D et al. (2015) Nutrition promotion approaches preferred by Australian adolescents attending schools in disadvantaged neighbourhoods: a qualitative study. BMC Pediatr 15, 61-73.

31. Selkie, EM, Benson M \& Moreno M (2011) Adolescents' views regarding uses of social networking websites and text messaging for adolescent sexual health education. Am J Health Educ 42, 205-212.

32. Gombert K, Douglas F, McArdle K et al (2017) Exploring the lives of vulnerable young people in relation to their food choices and practices. World J Educ 7, 50-61.

33. Backett-Millburn KC, Wills WJ, Roberts ML et al. (2010) Food and family practices: teenagers, eating and domestic life in differing socio-economic circumstances. Child Geogr 8, 303-314.

34. Peechey R \& Monsivais P (2015) Supermarket choice, shopping behaviour, SES and food purchase. Am J Prev Med $\mathbf{4 9}$, 868-877.

35. Lubans DR, Morgan PJ, Callister R et al. (2009) Effects of integrating pedometers, parental materials and E-mail support with an extracurricular school sport intervention. J Adolesc Health 44, 176-183.

36. Darmon N \& Drewnowski A (2015) Contribution of food prices and diet cost to socioeconomic disparities in diet quality and health: a systematic review. Nutr Rev 73, 643-660.

37. Caraher M, Lloyd S, Mansfield Met al. (2016) Secondary school pupils' food choices around schools in a London borough. Fast food and walls of crisps. Appetite 103, 208-220.

38. Jensen JD, Hartman H, Mul A et al. (2011) Economic incentives and nutritional behaviour of children in the school setting: a systematic review. Nutr Rev 69, 660-674.
39. Everson-Hock ES, Johnson M, Jones R et al. (2013) Community-based dietary and physical activity interventions in low socioeconomic groups in the UK: a mixed methods systematic review. Prev Med 56, 265-272.

40. Stevenson C, Doherty G, Barnett J et al. (2007) Adolescents' views of food and eating: identifying barriers to healthy eating. J Adolesc 3, 417-434.

41. Johnson S, Gray S \& Horrell A (2012) 'I want to look like that': healthism, the ideal body and physical education in a Scottish secondary school. Routledge Taylor and Francis Group. Discourse Stud Cult Politics Educ 34, 457-473.

42. Campbell K \& Peebles R (2014) Eating disorders in children and adolescents: State of the Art Review. Paediatrics 132, 582-592.

43. Neumark-Sztainer D, Flattum CF, Story M et al. (2008) Dietary approaches to healthy weight management for adolescents. Adolesc Med State Art Rev 19, 421-429.

44. Zotti F, Dalessandri D, Salgarello S et al. (2015) Usefulness of an app in improving oral hygiene compliance in adolescent orthodontic patients. Angle Orthod 86, 101-107.

45. Mauriello LM, Ciavatta MH \& Paiva AL (2010) Results of a multimedia multiple behaviour obesity prevention program for adolescents. Prev Med 51, 451-456.

46. O'Malley G, Dowdall G, Burls A et al. (2014) Exploring the usability of a mobile app for adolescent obesity management. JMIR Mhealth Uhealth 2, 29-39.

47. Dubuy V, De Cocker K, Bourdeaudhuij I et al. (2014) Evaluation of a real world intervention using professional footballer players to promote a healthy diet and physical activity in children and adolescents from a lower socioeconomic background: a controlled pre-test to post-test design. BMC Public Health 14, 457.

48. Michie S, Jochelson K \& Markham WA (2008) Low-Income Groups and Behaviour Change Interventions. A Review of the Intervention Content and Effectiveness. Kingsfund. https://www.kingsfund.org.uk/sites/files/kf/field/field_ document/low-income-groups-behaviour-change-intervention-kicking-bad-habits-paper.pdf (accessed March 2017).

49. Franklin VL, Waller A \& Pagliari C (2006) A randomized controlled trial of Sweet Talk, a text-messaging system to support young people with diabetes. Diabet Med 23, 1332-1338.

50. Shapiro JR, Bauer S, Hamer RM et al. (2008) Use of text messaging for monitoring sugar-sweetened beverages, physical activity and screen time in children: a pilot study. $J$ Nutr Educ Behav 40, 385-391.

51. Schoeppe S, Alley S, Rebar AL et al. (2017) Apps to improve diet, physical activity and sedentary behaviour in children and adolescents: a review of quality, features and behaviour change techniques. Int J Behav Nutr Phys Act 14, 83-93.

52. Spence S, White M, Adamson AJ et al. (2014) Does the use of passive or active consent affect consent of completion rates, or dietary data quality? Repeat cross-sectional survey among school children aged 11-12. BMJ Open 4, 1-6. 